\title{
Highly diastereoselective titanium(II)-mediated cyclizations of 1,7-(silyloxy)enynes
}

\author{
Nicole M. Barbour and Andrew J. Phillips* \\ Department of Chemistry and Biochemistry, University of Colorado, \\ Boulder, CO 80309-0215, USA \\ E-mail: andrew.phillips@,colorado.edu
}

\section{Dedicated to Professor Johann Mulzer on the occasion of his $65^{\text {th }}$ birthday}

\begin{abstract}
Cyclization of 1,7-(silyloxy)enynes with $\mathrm{Ti}(i-\mathrm{PrO})_{4}$ and $n$-BuLi yields 6-membered cyclic siloxanes in moderate to good yields. The reaction is highly diastereoselective (typical d.r.'s $>15: 1)$.
\end{abstract}

Keywords: Enyne, cyclization, silicon-tethered reactions, low-valent titanium, cyclic siloxanes

\section{Introduction}

We recently described the highly diastereoselective cyclization of 1,6-(silyloxy)enynes with $\mathrm{ClTi}(i-\mathrm{PrO})_{3}$ and Grignard reagents to give 5-membered cyclic siloxanes (Figure 1). ${ }^{1}$ Excision of the silicon gives rise to homoallylic alcohols, and these building blocks were used as part of the syntheses of dictyostatin ${ }^{2}$ and 7-demethylpericidin $\mathrm{A}_{1}{ }^{3}$. In conjunction with our interest in exploring the utility of these types of cyclization, we have examined the cyclization of 1,7(silyloxy)enynes (see also Figure 1), and in this paper we report our preliminary studies.

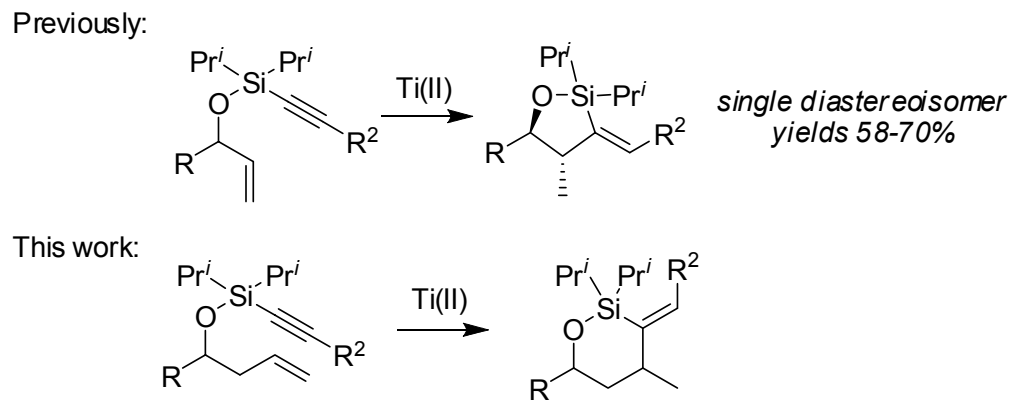

Figure 1. Cyclizations of 1,6- and 1,7-(silyloxy)enynes. 


\section{Results and Discussion}

Our initial studies focused on readily accessible 1,7-(silyloxy)enyne 2, which was prepared by silylation of alcohol $\mathbf{1}^{4}$ with bromodiisopropylhexynylsilane (Scheme 1). ${ }^{5}$ In our earlier work on the cyclization of 1,6-(silyloxy)enynes the combination of $\mathrm{ClTi}\left(\mathrm{OPr}^{i}\right)_{3}$ as the titanium source with $i-\mathrm{PrMgCl}$ (two equivalents with respect to the $\mathrm{Ti}$ ) at $-40{ }^{\circ} \mathrm{C}$ had proven optimal. Although these conditions resulted in cyclization of $\mathbf{2}$, there was a significant amount of unreacted starting material, and the isolated yields of $\mathbf{3}$ were never greater than $\sim 35 \%$. Although more starting material could be consumed by the addition of further equivalents of $\mathrm{ClTi}\left(\mathrm{OPr}^{i}\right)_{3}$ and the Grignard reagent after 3 hours, we viewed this approach as cumbersome and sub-optimal. Variation of temperature and solvents also failed to appreciably effect the levels of conversion and the combination of $\mathrm{Ti}\left(\mathrm{OPr}^{i}\right)_{4} / i-\mathrm{PrMgCl}$ was also fruitless.

Gratifyingly, turning to $n$ - $\mathrm{BuLi}$ as the reductant provided increased reactivity and in the case of 2, cyclization provided the desired cyclic siloxane 3 in $70 \%$ yield as essentially a single diastereoisomer (d.r. >15:1). NOESY NMR experiments showed a strong correlation between the hydrogen adjacent the alcohol and the hydrogen adjacent to the methyl group, which strongly suggested that the reaction occurs with 1,3-syn stereoinduction. Desilylation using TBAF provided the alcohol $\mathbf{4}$ in quantitative yield. ${ }^{6}$

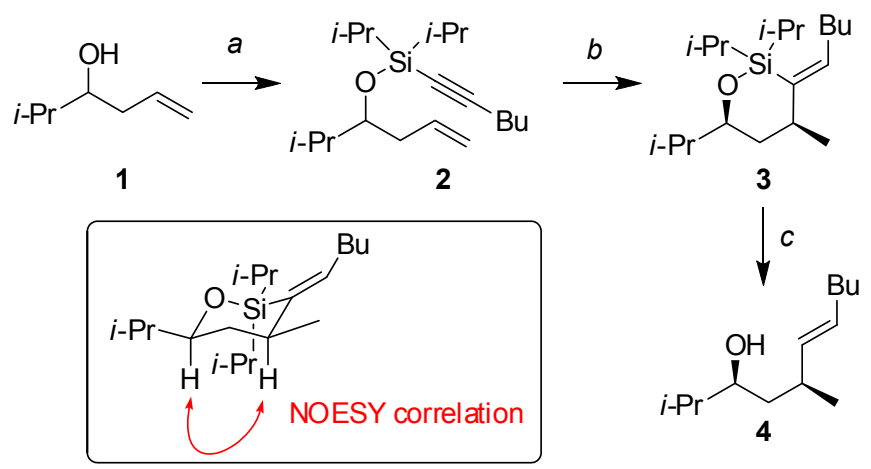

Scheme 1. Reagents and conditions: (a) bromodiisopropylhexynylsilane, DMAP, DMF, quantitative; (b) $\mathrm{Ti}(i-\mathrm{PrO})_{4}, n$-BuLi, THF, $-40{ }^{\circ} \mathrm{C} \rightarrow \mathrm{rt}, 70 \%$, d.r. $>15: 1$; (c) TBAF, DMF, $60{ }^{\circ} \mathrm{C}$ quant.

A variety of substrates that were cyclized and that provide some measure of the broad utility of the reaction are shown in Table 1. The reaction is highly diastereoselective with typical diastereoisomer ratios being $>15: 1$ as determined by ${ }^{1} \mathrm{H}$ NMR analysis of the crude reaction mixtures. ${ }^{7}$ Of particular note are substrates cyclized in entries 8 and 9, which produce stereochemical arrays that are difficult to access by alternative methods. In these cases the combination of anti- or syn- crotylation of an aldehyde, followed by (silyloxy)enyne cyclization provides a concise route to systems bearing adjacent methyl-group stereocenters. 
Table 1. Reagents and conditions: $\mathrm{Ti}(i-\mathrm{PrO})_{4}, n-\mathrm{BuLi}$, THF, $-40^{\circ} \mathrm{C} \rightarrow \mathrm{rt}$

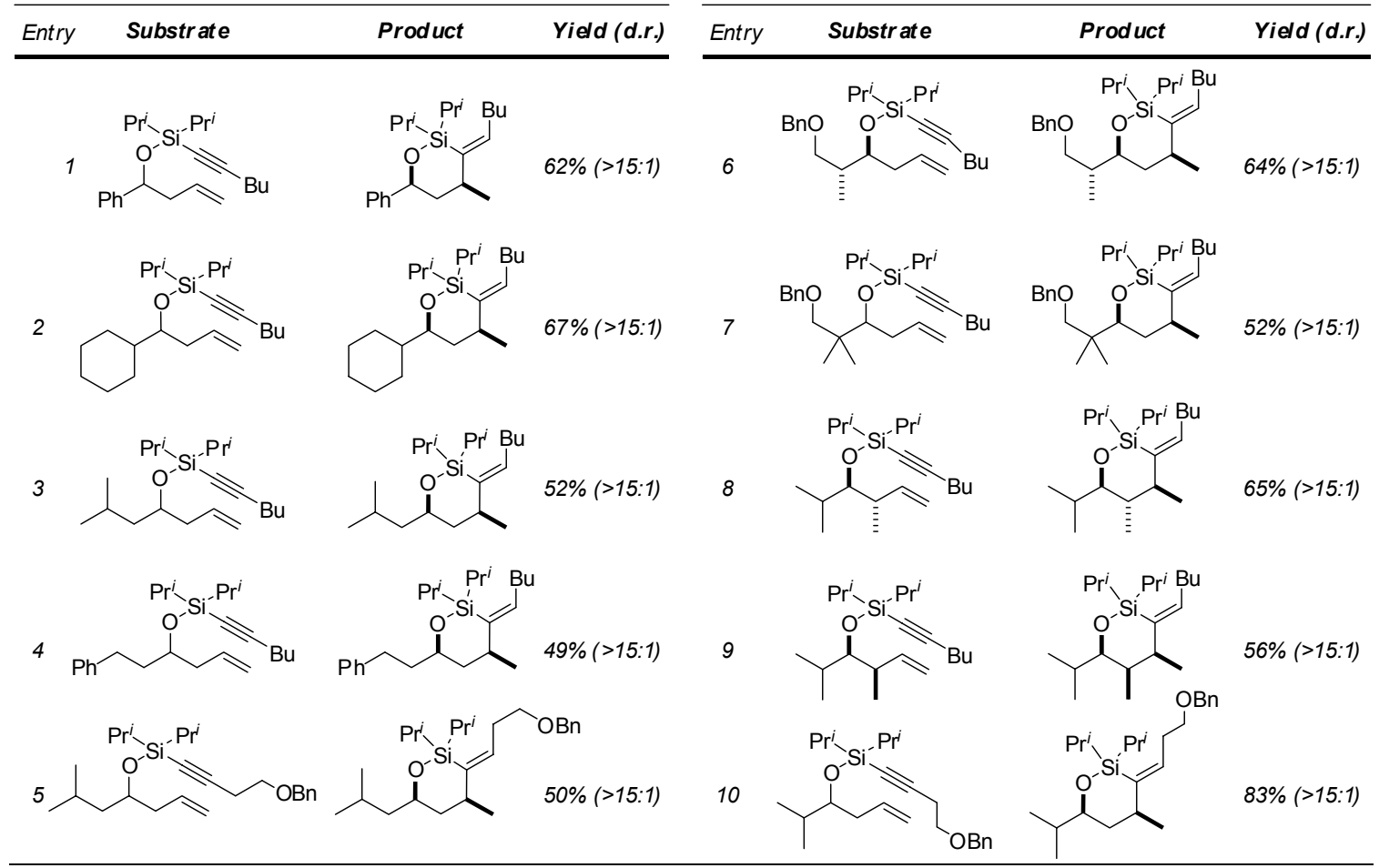

Although we have not performed extensive mechanistic studies, a simple mnemonic for the observed 1,3-syn stereoinduction can be proposed by employing a six membered chair-like transition state (Figure 2). In this model, the alkene orients itself to avoid a pseudo-1,3-diaxial interaction with an isopropyl group on silicon and cyclization occurs from conformer A.

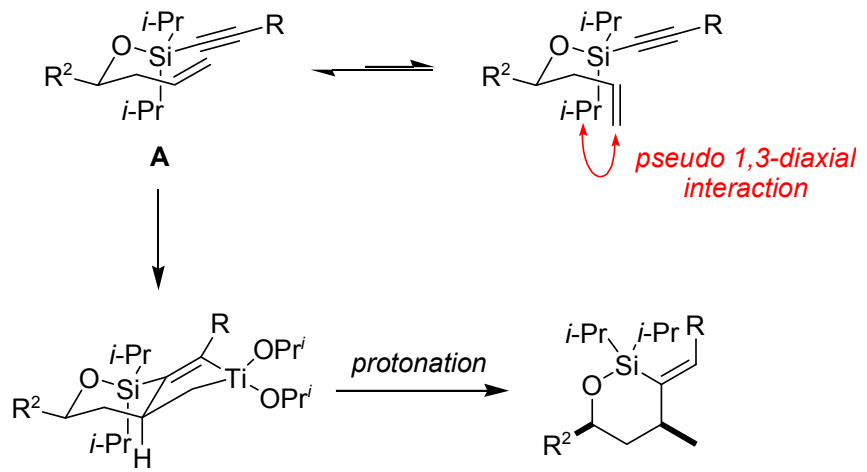

Figure 2. A simple model for the observed stereochemistry

In conclusion, we have described the cyclization of 1,7-(silyloxy)enynes to produce cyclic siloxanes. The reaction employs readily accessible reagents and provides high levels of 1,3-syn stereoinduction. Applications of this chemistry in the context of complex molecule synthesis are ongoing and will be reported in due course. 


\section{Experimental Section}

General Procedures. ${ }^{1} \mathrm{H}$ and ${ }^{13} \mathrm{C}$ spectra were recorded at $25{ }^{\circ} \mathrm{C}$ on a Varian Inova spectrometer operating at 500 and $100 \mathrm{MHz}$, respectively, using $\mathrm{CDCl}_{3}$ as the solvent and internal reference. Coupling constants are reported in Hertz, Hz. All non-aqueous reactions were run in flame-dried glassware under a dry $\mathrm{N}_{2}$ atmosphere. Toluene, THF, $\mathrm{CH}_{2} \mathrm{Cl}_{2}$, and $\mathrm{Et}_{2} \mathrm{O}$ were obtained from Aldrich (Pure-Pac) and further dried by passage through activated alumina. All flash chromatography was performed with normal phase silica gel (Silicycle, 35-75 $\mu \mathrm{m}$ particle size, $60 \AA ̊$ pore size).

\section{Representative procedure for the synthesis of a bromoalkynyl(diisopropyl)silane and} silylation of an alcohol. Hex-1-ynyl-diisopropyl-(1-isopropyl-but-3-enyloxy)-silane, 2

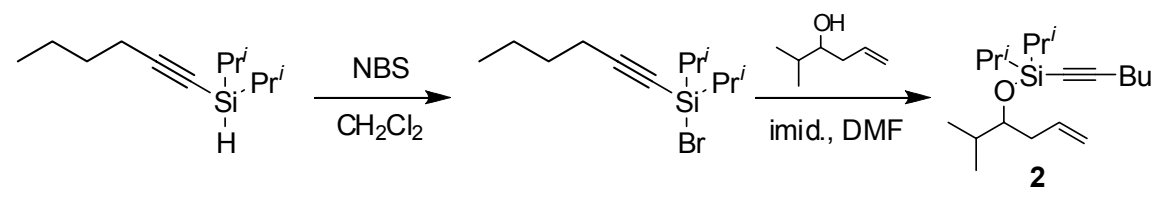

Hexynyl(diisopropyl)silane $(250 \mathrm{mg}, 1.27 \mathrm{mmol})$ in dichloromethane $(5 \mathrm{~mL})$ was cooled to $0{ }^{\circ} \mathrm{C}$, then $N$-bromosuccinimide $(277 \mathrm{mg}, 1.57 \mathrm{mmol}$ ) was added in a portionwise fashion. The cold bath was removed and the reaction was stirred for $90 \mathrm{~min}$ at room temperature. The solvent was removed on a rotary evaporator and the residue was diluted with pentane and filtered. The filtrate was evaporated and diluted with $d r y$ DMF $(2 \mathrm{~mL})$ and was then added to a separate flask containing the alcohol $(97 \mathrm{mg}, 0.85 \mathrm{mmol})$ and imidazole $(145 \mathrm{mg}, 2.13 \mathrm{mmol})$ in DMF (2.25 $\mathrm{mL}$ ). The reaction was stirred for $10 \mathrm{~h}$ at room temperature and was then diluted with ethyl acetate, washed with saturated aqueous $\mathrm{NH}_{4} \mathrm{Cl}$ solution and brine (4x), dried with $\mathrm{MgSO}_{4}$, filtered, and concentrated in vacuo. The residue was then purified by flash chromatography on silica gel eluting with $10 \% \mathrm{CH}_{2} \mathrm{Cl}_{2} / 90 \%$ hexanes to yield the 2 ( $246 \mathrm{mg}, 94 \%$ ).

Hex-1-ynyl-diisopropyl-(1-isopropyl-but-3-enyloxy)-silane(2).IR: 3074，2911，2169，1642, $1460 \mathrm{~cm}^{-1} ;{ }^{1} \mathrm{H}$ NMR $\left(500 \mathrm{MHz}, \mathrm{CDCl}_{3}\right): \delta 5.80(\mathrm{~m}, 1 \mathrm{H}), 5.00(\mathrm{~m}, 2 \mathrm{H}), 3.70(\mathrm{~m}, 1 \mathrm{H}), 2.24-2.32$ $(\mathrm{m}, 4 \mathrm{H}), 1.79-1.83(\mathrm{~m}, 1 \mathrm{H}), 1.43-1.56(\mathrm{~m}, 5 \mathrm{H}), 1.06-0.87(\mathrm{~m}, 22 \mathrm{H}) ;{ }^{13} \mathrm{C}$ NMR $(100 \mathrm{MHz}$, $\left.\mathrm{CDCl}_{3}\right): \delta 136.2,116.3,109.4,80.2,77.8,38.4,32.5,30.9,22.0,19.6,18.7,17.7,17.6,17.5$, 17.4, 17.3, 14.1, 13.8, 13.7; HRMS: calculated for $\mathrm{C}_{19} \mathrm{H}_{36} \mathrm{OSiNa}^{+}\left(\mathrm{M}+\mathrm{Na}^{+}\right) 331.2427$, found 331.2424 .

Hex-1-ynyl-diisopropyl-(1-phenyl-but-3-enyloxy)-silane, 4: IR: 2952, 2173, 1639, $1468 \mathrm{~cm}^{-1}$; ${ }^{1} \mathrm{H}$ NMR $\left(500 \mathrm{MHz}, \mathrm{CDCl}_{3}\right): \delta$ 7.34-7.23 (m, 5H), 5.82-5.74 (m, 1H), 5.03-4.96 (m, 3H), 2.60$2.49(\mathrm{~m}, 2 \mathrm{H}), 2.28-2.26(\mathrm{~m}, 2 \mathrm{H}), 1.58-1.43(\mathrm{~m}, 5 \mathrm{H}), 1.11-0.80(\mathrm{~m}, 16 \mathrm{H}) ;{ }^{13} \mathrm{C} \mathrm{NMR}(100 \mathrm{MHz}$, $\left.\mathrm{CDCl}_{3}\right): \delta 144.7,135.2,128.1,127.1,126.4,117,110.0,79.4,75.8,45.2,30.9,22.1,19.6,17.6$, 17.5, 17.3, 17.2, 13.8, 13.6, 13.3; HRMS: calculated for $\mathrm{C}_{22} \mathrm{H}_{34} \mathrm{OSiNa}^{+}\left(\mathrm{M}+\mathrm{Na}^{+}\right) 365.2271$, found 365.2273 . 
(1-Cyclohexyl-but-3-enyloxy)-hex-1-ynyl-diisopropyl-silane, 6: IR: 3070, 2919, 2846, 2169 , $1729 \mathrm{~cm}^{-1} ;{ }^{1} \mathrm{H}$ NMR $\left(500 \mathrm{MHz}, \mathrm{CDCl}_{3}\right): \delta$ 5.90-5.83 (m, 1H), 5.06-5.01 (m, 2H), 3.73-3.69 (m, $1 \mathrm{H}), 2.34-2.25(\mathrm{~m}, 4 \mathrm{H}), 1.65-1.72(\mathrm{~m}, 3 \mathrm{H}), 1.67-1.65(\mathrm{~m}, 2 \mathrm{H}), 1.56-1.43(\mathrm{~m}, 5 \mathrm{H}), 1.22-0.92(\mathrm{~m}$, $22 \mathrm{H}) ;{ }^{13} \mathrm{C}$ NMR $\left(100 \mathrm{MHz}, \mathrm{CDCl}_{3}\right): \delta 136.2,116.2,109.4,80.3,42.7,38.5,30.9,29.2,28.1$, 27.0, 26.7, 22.0, 19.7, 17.7, 17.6, 13.8, 13.7; HRMS: calculated for $\mathrm{C}_{22} \mathrm{H}_{40} \mathrm{OSiNa}{ }^{+}\left(\mathrm{M}+\mathrm{Na}^{+}\right)$ 371.2741 , found 371.2748 .

Hex-1-ynyl-(1-isobutyl-but-3-enyloxy)-diisopropyl-silane, 8: IR: 2956, 2866, $2173 \mathrm{~cm}^{-1} ;{ }^{1} \mathrm{H}$ NMR (500 MHz, $\left.\mathrm{CDCl}_{3}\right): \delta$ 7.29-7.18 (m, 5H), 5.91-5.84 (m, 1H), 5.11-5.06 (m, 2H), 4.04-4.02 $(\mathrm{m}, 1 \mathrm{H}), 2.80-2.75(\mathrm{~m}, 1 \mathrm{H}), 2.67-2.64(\mathrm{~m}, 1 \mathrm{H}), 2.42-2.37(\mathrm{~m}, 2 \mathrm{H}), 2.31-2.27(\mathrm{~m}, 2 \mathrm{H}), 1.90-1.80$ $(\mathrm{m}, 2 \mathrm{H}), 1.58-1.44(\mathrm{~m}, 6 \mathrm{H}), 1.10-0.93(\mathrm{~m}, 15 \mathrm{H}) ;{ }^{13} \mathrm{C}$ NMR $\left(100 \mathrm{MHz}, \mathrm{CDCl}_{3}\right): \delta 135.6,116.8$, 109.6, 79.9, 71.4, 46.0, 42.1, 30.9, 24.5, 23.4, 23.0, 22.1, 19.6, 17.6, 17.6, 13.8, 13.6; HRMS: calculated for $\mathrm{C}_{20} \mathrm{H}_{38} \mathrm{OSiNa}^{+}\left(\mathrm{M}+\mathrm{Na}^{+}\right)$345.2584, found 345.2584.

Hex-1-ynyl-diisopropyl-(1-phenethyl-but-3-enyloxy)-silane, 10: IR: 3058, 3021, 2935, 2866, 2169, 1639, 1602, $1464 \mathrm{~cm}^{-1} ;{ }^{1} \mathrm{H}$ NMR (500 MHz, $\left.\mathrm{CDCl}_{3}\right): \delta$ 7.27-7.16 (m, 5H), 5.89-5.84 (m, $1 \mathrm{H}), 5.09-5.04(\mathrm{~m}, 2 \mathrm{H}), 4.02-4.00(\mathrm{~m}, 1 \mathrm{H}), 2.75-2.73(\mathrm{~m}, 1 \mathrm{H}), 2.65-2.62(\mathrm{~m}, 1 \mathrm{H}), 2.40-2.35(\mathrm{~m}$, 2H), 2.29-2.25 (m, 2H), 1.88-1.78 (m, 2H), 1.56-1.42 (m, 6H), 1.08-0.91 (m, 15H); ${ }^{13} \mathrm{C}$ NMR $\left(100 \mathrm{MHz}_{\mathrm{CDCl}}\right): \delta 143.1,135.3,128.6,128.5,125.8,117.0,109.7,79.8,72.9,41.7,38.4$, $31.9,30.9,22.1,19.7,17.6,17.5,17.3,13.8,13.8,13.7,13.6$; HRMS: calculated for $\mathrm{C}_{24} \mathrm{H}_{38} \mathrm{OSiNa}^{+}\left(\mathrm{M}-\mathrm{Na}^{+}\right)$393.2584, found 393.2580.

(4-Benzyloxy-but-1-ynyl)-(1-isobutyl-but-3-enyloxy)-diisopropyl-silane, 12: IR: 3062, 3025, 2952, 2858, 2177, 1631, 1360, $1093 \mathrm{~cm}^{-1} ;{ }^{1} \mathrm{H} \mathrm{NMR}\left(500 \mathrm{MHz}, \mathrm{CDCl}_{3}\right): \delta$ 7.38-7.29 (m, 5H), 5.90-5.84 (m, 1H), 5.08-5.04 (m, 2H), $4.58(\mathrm{~s}, 2 \mathrm{H}), 4.02-4.00(\mathrm{~m}, 2 \mathrm{H}), 3.67-3.64(\mathrm{~m}, 2 \mathrm{H}), 2.63-$ $2.60(\mathrm{t}, 2 \mathrm{H}), 2.34-2.32(\mathrm{~m}, 2 \mathrm{H}), 1.78-1.75(\mathrm{~m}, 1 \mathrm{H}), 1.59-1.39(\mathrm{~m}, 1 \mathrm{H}), 1.33-1.28(\mathrm{~m}, 1 \mathrm{H}), 1.11-$ $1.05(\mathrm{~m}, 15 \mathrm{H}), 0.99-0.90(\mathrm{~m}, 9 \mathrm{H}) ;{ }^{13} \mathrm{C} \mathrm{NMR}\left(100 \mathrm{MHz}, \mathrm{CDCl}_{3}\right): \delta 138.3,135.5,128.6,127.9$, 127.9, 116.8, 105.6, 81.5, 73.3, 71.5, 68.8, 68.2, 46.0, 42.1, 24.5, 23.5, 23.0, 21.5, 17.6, 13.7, 13.6; HRMS: calculated for $\mathrm{C}_{25} \mathrm{H}_{40} \mathrm{O}_{2} \mathrm{SiNa}^{+}\left(\mathrm{M}+\mathrm{Na}^{+}\right)$423.2690, found 423.2687.

( \pm )-[1-(2-Benzyloxy-1-methyl-ethyl)-but-3-enyloxy]-hex-1-ynyl-diisopropyl-silane, 14: IR: 3066, 3029, 2895, 2165, 1636, 1464, 1354, 1243, 1080, 877, $685 \mathrm{~cm}^{-1}$; ${ }^{1} \mathrm{H}$ NMR (500 MHz, $\left.\mathrm{CDCl}_{3}\right): \delta 7.41-7.33(\mathrm{~m}, 5 \mathrm{H}), 6.00-5.96(\mathrm{~m}, 1 \mathrm{H}), 5.12-4.61(\mathrm{~m}, 2 \mathrm{H}), 4.59-4.06(\mathrm{~m}, 2 \mathrm{H}), 4.05-$ $3.67(\mathrm{~m}, 1 \mathrm{H}), 3.42-2.45(\mathrm{~m}, 1 \mathrm{H}), 2.44-2.37(\mathrm{~m}, 2 \mathrm{H}), 2.32-2.30(\mathrm{~m}, 2 \mathrm{H}), 1.59-1.49(\mathrm{~m}, 4 \mathrm{H}), 1.18-$ $0.99(\mathrm{~m}, 25 \mathrm{H}) ;{ }^{13} \mathrm{C} \mathrm{NMR}\left(100 \mathrm{MHz}, \mathrm{CDCl}_{3}\right): \delta 139.2,135.7,128.5,127.7,127.6,116.8,109.6$, 80.1, 74.9, 73.1, 72.8, 38.4, 30.9, 22.1, 19.7, 17.7, 13.8; HRMS: calculated for $\mathrm{C}_{26} \mathrm{H}_{42} \mathrm{O}_{2} \mathrm{SiNa}^{+}$ $\left(\mathrm{M}+\mathrm{Na}^{+}\right)$437.2846, found 437.2849.

[1-(2-Benzyloxy-1,1-dimethyl-ethyl)-but-3-enyloxy]-hex-1-ynyl-diisopropyl-silane, 16: IR: 3070, 3021, 2944, 2866, 2169, 1460, 1370, 1084, 877, 734, $591 \mathrm{~cm}^{-1} ;{ }^{1} \mathrm{H}$ NMR (500 MHz, $\left.\mathrm{CDCl}_{3}\right): \delta 7.36-7.23(\mathrm{~m}, 5 \mathrm{H}), 6.05-6.00(\mathrm{~m}, 1 \mathrm{H}), 5.05-4.97(\mathrm{~m}, 2 \mathrm{H}), 4.50(\mathrm{q}, 2 \mathrm{H}), 3.94-3.92(\mathrm{q}$, $1 \mathrm{H}), 3.30(\mathrm{q}, 2 \mathrm{H}), 2.49-2.45(\mathrm{~m}, 1 \mathrm{H}), 2.33-2.30(\mathrm{~m}, 1 \mathrm{H}), 2.27-2.24(\mathrm{t}, 2 \mathrm{H}), 1.54-1.43(\mathrm{~m}, 5 \mathrm{H})$, 1.21-0.91 (m, 30H); ${ }^{13} \mathrm{C}$ NMR (100 MHz, $\left.\mathrm{CDCl}_{3}\right): \delta 139.4,137.9,128.4,127.6,127.5,115.6$, $109.4,80.8,73.3,40.8,38.2,30.9,22.1,21.9,21.4,19.7,18.0,17.9,17.7,17.5,17.4,14.1,14.0$, 13.9, 13.8; HRMS: calculated for $\mathrm{C}_{27} \mathrm{H}_{44} \mathrm{O}_{2} \mathrm{SiNa}^{+}\left(\mathrm{M}-\mathrm{Na}^{+}\right)$451.3002, found 451.2487. 
(3R,4S)-Hex-1-ynyl-diisopropyl-(1-isopropyl-2-methyl-but-3-enyloxy)-silane, 18: IR: 3078 , 2964, 2862, 2165, 1645, 1635, 1464, 1382, $1243 \mathrm{~cm}^{-1} ;{ }^{1} \mathrm{H}$ NMR $\left(500 \mathrm{MHz}, \mathrm{CDCl}_{3}\right): \delta 6.01-5.94$ $(\mathrm{m}, 1 \mathrm{H}), 5.03-4.97(\mathrm{~m}, 2 \mathrm{H}), 3.60-3.58(\mathrm{~m}, 1 \mathrm{H}), 2.51-2.49(\mathrm{~m}, 1 \mathrm{H}), 2.30-2.25(\mathrm{~m}, 2 \mathrm{H}), 1.87-1.81$ $(\mathrm{m}, 1 \mathrm{H}), 1.56-1.46(\mathrm{~m}, 5 \mathrm{H}), 1.11-0.88(\mathrm{~m}, 25 \mathrm{H}) ;{ }^{13} \mathrm{C}$ NMR $\left(100 \mathrm{MHz}, \mathrm{CDCl}_{3}\right): \delta$ 142.2, 113.6, 109.3, 77.2, 76.9, 42.3, 32.3, 30.9, 22.0, 20.1, 19.6, 19.0, 17.9, 17.8, 17.7, 17.6, 17.3, 14.1, 14.0, 13.9, 13.7; HRMS: calculated for $\mathrm{C}_{20} \mathrm{H}_{38} \mathrm{OSiNa}^{+}\left(\mathrm{M}+\mathrm{Na}^{+}\right)$345.2584, found 345.2576.

(3R,4R)-Hex-1-ynyl-diisopropyl-(1-isopropyl-2-methyl-but-3-enyloxy)-silane, 20: IR: 3074, 2895, 2711, 2356, 2328, 2165, 1639, 1460, 1378, 1064, 872, $677 \mathrm{~cm}^{-1}$; ${ }^{1} \mathrm{H}$ NMR (500 MHz, $\left.\mathrm{CDCl}_{3}\right): \delta 5.92-5.87(\mathrm{~m}, 1 \mathrm{H}), 5.02-4.94(\mathrm{~m}, 2 \mathrm{H}), 3.58-3.56(\mathrm{~m}, 1 \mathrm{H}), 2.42(\mathrm{q}, 1 \mathrm{H}), 2.28-2.24(\mathrm{~m}$, $3 \mathrm{H}), 1.84-1.83(\mathrm{~m}, 1 \mathrm{H}), 1.54-1.43(\mathrm{~m}, 6 \mathrm{H}), 1.09-0.86(\mathrm{~m}, 23 \mathrm{H}) ;{ }^{13} \mathrm{C} \mathrm{NMR}\left(100 \mathrm{MHz}, \mathrm{CDCl}_{3}\right): \delta$ $142.9,113.2,109.3,81.9,80.3,42.4,32.0,30.9,22.0,20.4,19.6,17.9,17.8,17.7,17.6,17.4$, 17.3, 16.5, 14.1, 14.0, 13.8, 13.7; HRMS: calculated for $\mathrm{C}_{20} \mathrm{H}_{38} \mathrm{OSiNa}^{+}\left(\mathrm{M}+\mathrm{Na}^{+}\right) 345.2584$, found 345.2584 .

4-Benzyloxy-but-1-ynyl)-diisopropyl-(1-isopropyl-but-3-enyloxy)-silane, 22: IR: 3433, 3066, 3021, 2866, 2169, 1704, 1468, 1362, $1093 \mathrm{~cm}^{-1}$; ${ }^{1} \mathbf{H}$ NMR (500 MHz, $\left.\mathrm{CDCl}_{3}\right): \delta$ 7.41-7.29 (m, $5 \mathrm{H}), 5.93-5.87(\mathrm{~m}, 1 \mathrm{H}), 5.10-5.03(\mathrm{~m}, 1 \mathrm{H}), 4.61-4.58(\mathrm{~m}, 2 \mathrm{H}), 3.66(\mathrm{q}, 2 \mathrm{H}), 2.61(\mathrm{q}, 2 \mathrm{H}), 2.35-$ $2.31(\mathrm{~m}, 2 \mathrm{H}), 1.86-1.83(\mathrm{~m}, 1 \mathrm{H}), 1.13-0.91(\mathrm{~m}, 26 \mathrm{H}) ;{ }^{13} \mathbf{C}$ NMR $\left(100 \mathrm{MHz}, \mathrm{CDCl}_{3}\right): \delta 138.3$, $138.3,136.1,128.7,128.6,127.9,116.4,105.5,81.2,77.8,73.3,73.2,68.9,68.6,38.5,32.5$, $21.5,21.4,19.0,18.7,17.8,17.7,17.6,17.5,17.3,17.3,17.1,13.8,13.7,13.3$; HRMS: calculated for $\mathrm{C}_{24} \mathrm{H}_{38} \mathrm{O}_{2} \mathrm{SiNa}^{+}\left(\mathrm{M}+\mathrm{Na}^{+}\right)$409.2533, found 409.2531.

\section{Representative procedure for the enyne cyclization. 2,2,6-Triisopropyl-4-methyl-3-} pentylidene-[1,2]oxasilinane, 3

A solution of $\operatorname{Ti}\left(\mathrm{OPr}^{\mathrm{i}}\right)_{4}(0.364 \mathrm{~mL}, 1.21 \mathrm{mmol})$ in THF $(2.2 \mathrm{~mL})$ was cooled to $-78{ }^{\circ} \mathrm{C}$, then $n$ BuLi (1.60 mL, 1.6 M, $2.56 \mathrm{mmol})$ was added. This solution was stirred $15 \mathrm{~min}$, then warmed to $0{ }^{\circ} \mathrm{C}$ and the dark red colored solution was stirred for $20 \mathrm{~min}$. The reaction was then cooled to $40{ }^{\circ} \mathrm{C}$ and the substrate alcohol 2 was added to the reaction as a solution in THF. This was warmed to room temperature over $10 \mathrm{~h}$. This was quenched with $\mathrm{NH}_{4} \mathrm{Cl}$, diluted with ethyl acetate, washed with brine, dried with $\mathrm{MgSO}_{4}$, filtered, and concentrated in vacuo. The residue was purified by flash chromatography on silica gel eluting with $10 \% \mathrm{CH}_{2} \mathrm{Cl}_{2} / 90 \%$ hexanes to yield the siloxane $(90.2 \mathrm{mg}, 62 \%)$.

2,2,6-Triisopropyl-4-methyl-3-pentylidene-[1,2]oxasilinane, 2: IR: 2829, 2169, $1610 \mathrm{~cm}^{-1} ;{ }^{1} \mathrm{H}$ NMR (500 MHz, $\left.\mathrm{CDCl}_{3}\right): \delta$ 5.99-5.96 (m, 1H), $3.65(\mathrm{~m}, 1 \mathrm{H}), 2.33-2.29(\mathrm{~m}, 1 \mathrm{H}), 2.06-1.98(\mathrm{~m}$, 2H), 1.60-1.54 (m, 2H), 1.38-1.31 (m, 5H), 1.26-0.86 (m, 22H); ${ }^{13} \mathrm{C}$ NMR (100 MHz, CDCl $): \delta$ 138.8, 138.1, 79.9, 41.4, 38.3, 35.4, 33.2, 32.6, 22.9, 20.6, 19.0, 18.2, 18.1, 18.0, 17.5, 15.3, 14.3, 14.3; HRMS: calculated for $\mathrm{C}_{19} \mathrm{H}_{38} \mathrm{OSiNa}^{+}\left(\mathrm{M}+\mathrm{Na}^{+}\right)$333.2583, found 333.2587.

2,2-Diisopropyl-4-methyl-3-pentylidene-6-phenyl-[1,2]oxasilinane, 5 (Table 1, Entry 1): IR: 2956, 2854, $1464 \mathrm{~cm}^{-1} ;{ }^{1} \mathrm{H}$ NMR (500 MHz, $\left.\mathrm{CDCl}_{3}\right): \delta$ 7.40-7.22 (m, 5H), 6.07-6.04 (m, 1H), 5.08-5.06 (m, 1H), 2.58-2.55 (m, 1H), 2.12-2.04 (m, 2H), 1.85-1.82 (m, 1H), 1.43-1.34 (m, 5H), 
1.26-0.91 (m, 20H); ${ }^{13} \mathrm{C}$ NMR (100 MHz, $\left.\mathrm{CDCl}_{3}\right): \delta 146.3,139.1,137.5,128.3,136.9,125.4$, 47.9, 39.8, 38.8, 33.3, 32.6, 22.9, 20.4, 18.2, 18.0, 17.6, 15.4, 14.3; HRMS: calculated for $\mathrm{C}_{22} \mathrm{H}_{36} \mathrm{OSiNa}^{+}\left(\mathrm{M}+\mathrm{Na}^{+}\right) 367.2428$, found 367.2431 .

6-Cyclohexyl-2,2-diisopropyl-4-methyl-3-pentylidene-[1,2]oxasilinane, 7 (Table 1, Entry 2): IR: 2948, $2854 \mathrm{~cm}^{-1}$; ${ }^{1} \mathrm{H}$ NMR $\left(500 \mathrm{MHz}, \mathrm{CDCl}_{3}\right): \delta 5.99-5.97(\mathrm{~m}, 1 \mathrm{H}), 3.67-3.64(\mathrm{~m}, 1 \mathrm{H}), 2.32-$ $2.29(\mathrm{~m}, 1 \mathrm{H}), 2.07-2.00(\mathrm{~m}, 2 \mathrm{H}), 1.89-1.86(\mathrm{~m}, 1 \mathrm{H}), 1.75-1.74(\mathrm{~m}, 2 \mathrm{H}), 1.67-1.65(\mathrm{~m}, 2 \mathrm{H}), 1.58-$ $1.55(\mathrm{~m}, 1 \mathrm{H}), 1.39-0.92(\mathrm{~m}, 27 \mathrm{H}) ;{ }^{13} \mathrm{C} \mathrm{NMR}\left(100 \mathrm{MHz}, \mathrm{CDCl}_{3}\right): \delta 138.9,138.1,79.4,45.5,41.4$, 38.3, 33.3, 32.6, 29.3, 28.5, 27.0, 26.7, 26.6, 22.9, 20.6, 18.2, 18.1, 17.5, 15.3, 14.3; HRMS: calculated for $\mathrm{C}_{22} \mathrm{H}_{42} \mathrm{OSiNa}^{+}\left(\mathrm{M}+\mathrm{Na}^{+}\right)$373.2897, found 373.2903.

6-Isobutyl-2,2-diisopropyl-4-methyl-3-pentylidene-[1,2] oxasilinane, 9 (Table 1, Entry 3): IR: 2952, 2862, 1733, $1615 \mathrm{~cm}^{-1} ;{ }^{1} \mathrm{H}$ NMR $\left(500 \mathrm{MHz}, \mathrm{CDCl}_{3}\right): \delta$ 5.98-5.95 $(\mathrm{m}, 1 \mathrm{H}), 3.99-3.96(\mathrm{~m}$, $1 \mathrm{H}), 2.34-2.32(\mathrm{~m}, 1 \mathrm{H}), 2.05-1.98(\mathrm{~m}, 2 \mathrm{H}), 1.88-1.82(\mathrm{~m}, 1 \mathrm{H}), 1.56-1.53(\mathrm{~m}, 1 \mathrm{H}), 1.40-1.31(\mathrm{~m}$, 7H), 1.15-0.88 (m, 26H); ${ }^{13} \mathrm{C}$ NMR (100 MHz, $\left.\mathrm{CDCl}_{3}\right): \delta$ 138.9, 138.0, 73.0, 48.5, 45.1, 38.0, 33.2, 32.6, 24.6, 23.7, 22.9, 22.5, 20.4, 18.2, 18.1, 18.0, 17.5, 15.2, 14.3, 14.1; HRMS: calculated for $\mathrm{C}_{20} \mathrm{H}_{40} \mathrm{OSiNa}{ }^{+}\left(\mathrm{M}+\mathrm{Na}^{+}\right) 347.2741$, found 347.2746 .

2,2-Diisopropyl-4-methyl-3-pentylidene-6-phenethyl-[1,2]oxasilinane, 11 (Table 1, Entry 4): IR: 2931, 2862, 2165, 1606, $1456 \mathrm{~cm}^{-1} ;{ }^{1} \mathrm{H}$ NMR $\left(500 \mathrm{MHz}, \mathrm{CDCl}_{3}\right): \delta$ 7.33-7.20 (m, 5H), 6.01$6.00(\mathrm{~m}, 1 \mathrm{H}), 3.96-3.91(\mathrm{~m}, 1 \mathrm{H}), 2.86-2.83(\mathrm{~m}, 1 \mathrm{H}), 2.77-2.71(\mathrm{~m}, 1 \mathrm{H}), 2.37-2.33(\mathrm{~m}, 1 \mathrm{H}), 2.11-$ $2.02(\mathrm{~m}, 2 \mathrm{H}), 1.77-1.71(\mathrm{~m}, 2 \mathrm{H}), 1.61-1.59(\mathrm{~m}, 1 \mathrm{H}), 1.43-1.35(\mathrm{~m}, 5 \mathrm{H}), 1.13-0.94(\mathrm{~m}, 20 \mathrm{H}) ;{ }^{13} \mathrm{C}$ NMR (100 MHz, $\left.\mathrm{CDCl}_{3}\right): \delta 143.1,138.7,138.1,128.9,128.5,126.8,73.8,44.6,41.2,37.9,33.3$, 32.6, 32.0, 22.9, 20.4, 18.2, 18.1, 17.5, 15.3, 14.4, 14.1; HRMS: calculated for $\mathrm{C}_{24} \mathrm{H}_{40} \mathrm{OSiNa}^{+}$ $\left(\mathrm{M}+\mathrm{Na}^{+}\right)$395.2741, found 395.2755.

3-(3-Benzyloxy-propylidene)-6-isobutyl-2,2-diisopropyl-4-methyl-[1,2]oxasilinane, 13 (Table 1, Entry 5): IR: 2952.2, 2858, 1464, 1358, 1101, 1064, $987 \mathrm{~cm}^{-1} ;{ }^{1} \mathrm{H}$ NMR $\left(500 \mathrm{MHz}, \mathrm{CDCl}_{3}\right): \delta$ 7.39-7.29 (m, 5H), 6.02-6.0 (m, $1 \mathrm{H}), 4.56-4.55(\mathrm{~s}, 2 \mathrm{H}), 4.02-0.01(\mathrm{~m}, 1 \mathrm{H}), 3.54-3.51(\mathrm{t}, 2 \mathrm{H})$, 2.45-2.37 (m, 3H), 1.90-1.87 (m, 1H), 1.59-1.56 (m, $1 \mathrm{H}), 1.47-1.39(\mathrm{~m}, 2 \mathrm{H}), 1.18-0.90(\mathrm{~m}$, $28 \mathrm{H}) ;{ }^{13} \mathrm{C}$ NMR $\left(100 \mathrm{MHz}, \mathrm{CDCl}_{3}\right): \delta 141.9,138.7,133.1,128.6,127.8,127.8,73.1,73.0,70.3$, 48.5, 45.0, 38.2, 33.9, 23.6, 23.7, 22.5, 20.3, 18.1, 18.0, 17.8, 17.6, 17.5, 15.2, 14.0; HRMS: calculated for $\mathrm{C}_{25} \mathrm{H}_{42} \mathrm{O}_{2} \mathrm{SiNa}^{+}\left(\mathrm{M}+\mathrm{Na}^{+}\right)$425.2846, found 425.2848.

6-(2-Benzyloxy-1-methyl-ethyl)-2,2-diisopropyl-4-methyl-3-pentylidene-[1,2]oxasilinane, 15 (Table 1, Entry 6): IR: 3062, 3029, 2948, 1941, 1859, 1802, 1602, 1455, 1370, 1101, 877, 779, $709 \mathrm{~cm}^{-1} ;{ }^{1} \mathrm{H}$ NMR $\left(500 \mathrm{MHz}, \mathrm{CDCl}_{3}\right): \delta$ 7.41-7.29 (m, 5H), 6.06-6.03 (m, 1H), $4.57(\mathrm{~s}, 2 \mathrm{H})$, 3.92-3.89 (m, 1H), 3.71-3.69 (m, 1H), 3.50-3.47 (m, 1H), $2.37(\mathrm{~m}, 1 \mathrm{H}), 2.10-2.05(\mathrm{~m}, 2 \mathrm{H}), 1.88-$ $1.85(\mathrm{~m}, 1 \mathrm{H}), 1.69-1.67(\mathrm{~d}, 1 \mathrm{H}), 1.43-0.97(\mathrm{~m}, 28 \mathrm{H}) ;{ }^{13} \mathrm{C} \mathrm{NMR}\left(100 \mathrm{MHz}, \mathrm{CDCl}_{3}\right): \delta 139.3$, 138.7, 138.3, 128.5, 127.8, 127.6, 73.3, 72.8, 41.5, 41.4, 38.2, 33.3, 32.6, 22.9, 20.6, 18.2, 18.1, 17.6, 15.3, 14.4, 14.2; HRMS: calculated for $\mathrm{C}_{26} \mathrm{H}_{44} \mathrm{O}_{2} \mathrm{SiNa}^{+}\left(\mathrm{M}+\mathrm{Na}^{+}\right)$439.3003, found 439.3004 .

6-(2-Benzyloxy-1,1-dimethyl-ethyl)-2,2-diisopropyl-4-methyl-3-pentylidene-

[1,2]oxasilinane, 17 (Table 1, Entry 7): IR: 3066, 3029, 2952, 2923, 2858, 2169, 1455, 1374, $1097,1019 \mathrm{~cm}^{-1} ;{ }^{1} \mathrm{H}$ NMR (500 MHz, $\left.\mathrm{CDCl}_{3}\right): \delta 7.37-7.29(\mathrm{~m}, 5 \mathrm{H}), 6.02-6.00(\mathrm{~m}, 1 \mathrm{H}), 4.53(\mathrm{q}$, 
$2 \mathrm{H}), 3.90(\mathrm{~d}, 1 \mathrm{H}), 3.45(\mathrm{~d}, 1 \mathrm{H}), 3.20(\mathrm{~d}, 1 \mathrm{H}), 2.34-2.25(\mathrm{~m}, 2 \mathrm{H}), 2.07-2.01(\mathrm{~m}, 2 \mathrm{H}), 1.56(\mathrm{~d}, 2 \mathrm{H})$, 1.41-0.78 (m, 29H); ${ }^{13} \mathrm{C}$ NMR (100 MHz, $\left.\mathrm{CDCl}_{3}\right): \delta 139.3,138.5,138.4,128.4,127.6,127.5$, $127.4,78.7,73.5,60.5,39.9,38.7,38.2$, 33.2, 32.6, 30.5, 30.0, 22.9, 21.9, 21.2, 20.8, 20.1, 18.2, 18.1, 17.7, 17.6, 17.5, 17.4, 17.3, 15.4, 14.4, 14.3; HRMS: calculated for $\mathrm{C}_{27} \mathrm{H}_{46} \mathrm{O}_{2} \mathrm{SiNa}^{+}$ $\left(\mathrm{M}+\mathrm{Na}^{+}\right) 453.3159$, found 453.3175 .

(4S,5S,6R)-2,2,6-Triisopropyl-4,5-dimethyl-3-pentylidene-[1,2]oxasilinane, 19 (Table 1, Entry 8): IR: 2952, 2862, 1741, 1606, 1460, 1378, 1243, 1048, $901 \mathrm{~cm}^{-1} ;{ }^{1} \mathrm{H}$ NMR (500 MHz, $\left.\mathrm{CDCl}_{3}\right): \delta 6.06-6.03(\mathrm{~m}, 1 \mathrm{H}), 3.32(\mathrm{~m}, 1 \mathrm{H}), 2.09-2.03(\mathrm{~m}, 4 \mathrm{H}), 1.82-1.79(\mathrm{~m}, 1 \mathrm{H}), 1.37-1.27(\mathrm{~m}$, 14H), 1.12-0.91 (m, 19H); ${ }^{13} \mathrm{C}$ NMR (100 MHz, $\left.\mathrm{CDCl}_{3}\right) ; \delta 142.1,138.4,82.6,46.2,41.7,33.5$, 32.5, 31.4, 30.0, 22.9, 21.6, 20.8, 18.6, 18.4, 18.3, 18.2, 18.1, 17.8, 17.6, 17.2, 15.3, 14.5, 14.3; HRMS: calculated for $\mathrm{C}_{20} \mathrm{H}_{40} \mathrm{OSiNa}^{+}\left(\mathrm{M}+\mathrm{Na}^{+}\right)$347.2741, found 347.2747.

(4S,5R,6R)-2,2,6-Triisopropyl-4,5-dimethyl-3-pentylidene-[1,2]oxasilinane, 21 (Table 1, Entry 9): IR: $2960,2915,2858,1460,1382,1117,991 \mathrm{~cm}^{-1} ;{ }^{1} \mathbf{H}$ NMR $\left(500 \mathrm{MHz}, \mathrm{CDCl}_{3}\right): \delta$ 5.89-5.87 (m, 1H), 3.44-3.42 (m, 1H), 2.51-2.50 (m, 1H), 2.19-2.01 (m, 2H), 1.72-1.60 (m, 2H), 1.40-1.27 (m, 7H), 1.12-0.64 (m, $\left.26 \mathrm{H}) ;{ }^{13} \mathbf{C ~ N M R ~ ( 1 0 0 ~ M H z , ~} \mathrm{CDCl}_{3}\right) ; \delta$ 139.1, 136.2, 84.6, 43.9, 39.8, 33.3, 32.8, 32.3, 22.9, 20.4, 18.8, 18.4, 18.3, 18.2, 17.6, 14.9, 14.5, 14.3, 5.8; HRMS: calculated for $\mathrm{C}_{20} \mathrm{H}_{40} \mathrm{OSiNa}^{+}\left(\mathrm{M}+\mathrm{Na}^{+}\right) 347.2742$, found 347.2770.

3-(3-Benzyloxy-propylidene)-2,2,6-triisopropyl-4-methyl-[1,2]oxasilinane, 23 (Table 1, Entry 10): IR: 2964, 2862, 1468, 1101, $1052 \mathrm{~cm}^{-1} ;{ }^{1} \mathrm{H} \mathrm{NMR}\left(500 \mathrm{MHz}, \mathrm{CDCl}_{3}\right): \delta$ 7.39-7.29 (m, 5H), 6.04-6.02 (m, 1H), $4.56(\mathrm{~s}, 2 \mathrm{H}), 3.70-3.67(\mathrm{~m}, 1 \mathrm{H}), 3.55-3.52(\mathrm{~m}, 2 \mathrm{H}), 2.45-2.32(\mathrm{~m}, 4 \mathrm{H}), 1.64-$ $1.58(\mathrm{~m}, 2 \mathrm{H}), 1.48(\mathrm{~s}, 1 \mathrm{H}), 1.31(\mathrm{~s}, 1 \mathrm{H}), 1.16-0.89(\mathrm{~m}, 38 \mathrm{H}) ;{ }^{13} \mathrm{C} \mathrm{NMR}\left(100 \mathrm{MHz}, \mathrm{CDCl}_{3}\right): \delta$ $141.9,138.8,133.2,128.6,127.8,127.8,79.9,73.2,70.3,41.3,38.5,35.4,33.9,20.6,19.0 .18 .2$, 18.1, 18.0, 17.4, 15.4, 14.2; HRMS: calculated for $\mathrm{C}_{24} \mathrm{H}_{40} \mathrm{NaO}_{2} \mathrm{SiNa}^{+}\left(\mathrm{M}+\mathrm{Na}^{+}\right) 411.2690$, found 411.2699 .

\section{Acknowledgements}

This research was supported by the National Science Foundation CAREER Program (Award \# 0645787).

\section{References}

1. O’Neil, G. W.; Phillips, A. J. Tetrahedron Lett. 2004, 45, 4253

2. O’Neil, G. W.; Phillips, A. J. J. Am. Chem. Soc. 2006, 128, 5340.

3. Keaton, K. A.; Phillips, A. J. J. Am. Chem. Soc. 2006, 128, 408.

4. Readily prepared by Barbier-type allylation using allyl bromide and zinc. See Hoffman, R.; Brueckner, R.; Chem. Ber. 1992, 125, 2731. 
5. A variety of functionalized bromodiisopropylalkynylsilanes are readily accessible by alkylation of lithioacetylides with chlorodiisopropylsilane. Subsequent bromination and reaction with an alcohol provides the (silyloxy)enyne. See the experimental section for a representative procedure.

6. Ozonolysis of this alkene and reduction of the aldehyde provided a diol for which data could be compared to the known 2,4-anti-2,5-dimethylhexane-1,4-diol. See: Still, W. C.; Darst, K. P. J. Am. Chem. Soc. 1980, 102, 7385.

7. The stereochemistry of the examples in Table 1 was assigned by analogy to that for 3 . 